\title{
Werner Jaegers Blicke auf das antike Christentum
}

Auf den ersten Blick ist zu befürchten, dass mein Beitrag eine schlichte Dublette darstellt - wobei ich in unseren Zusammenhängen das Drama natürlich vornehmer formulieren könnte: Es ist zu befürchten, dass meine Ausführungen bestenfalls eine $\pi \alpha \lambda ı \nu \omega \delta i ́ \alpha$ werden können. Paul T. Keyser, heute als Informatiker tätig, hat auf der Konferenz „Werner Jaeger reconsidered“ von 1990 über „Werner Jaeger's Early Christianity and Greek Paideia“ gehandelt, ${ }^{1}$ aber in diesem Beitrag weit mehr besprochen als die Carl Newell Jackson-Vorlesungen, die Jaeger 1960 unter eben diesem Titel in Harvard gehalten hat und die 1963 unter dem Titel „Das frühe Christentum und die griechische Bildung“ im Verlag Walter de Gruyter in Berlin erschienen sind. ${ }^{2}$ Das schmale, aber gehaltvolle Büchlein war Jaegers letzte Veröffentlichung, am 19. Oktober 1961 ist er gestorben; wir werden auf den Text am Ende dieser Ausführungen zurückkommen.

Eine schlichte Repetition der Ausführungen von Keyser oder eben eine $\pi \alpha \lambda ı \omega \delta \delta^{\prime} \alpha$ muss freilich nicht befürchtet werden, weil doch für ein Gesamtbild allerlei weitere Quellen heranzuziehen sind, die im genannten Beitrag fehlen, und zudem alles stärker im Lichte der Geschichte der Beschäftigung mit dem antiken Christentum in der ersten Hälfte des zwanzigsten Jahrhunderts gesehen werden muss. Hinzu kommt das Problem fehlender Quellen: Keyser hat nicht nur Äußerungen Jaegers über das antike Christentum aus diversen Lebensepochen und literarischen Kontexten zusammengestellt, sondern auch nach deren Wurzeln in Jaegers religiöser Mentalität aus Kinder- und Jugendtagen gesucht. ${ }^{3}$ Freilich muss man sich klarmachen, dass die Quellen, auf die sich Keyser bezieht, doch relativ spät zu datieren sind: In einem „Zur Einführung“ überschriebenen Vorwort für seine beiden Bände der „Scripta Minora“ von 1960 hat Jaeger mit knappen Worten das Kempener Gymnasium Thomaeum charakterisiert, an das er im Jahre 1902 nach vier Jahren auf der katholischen höheren Knabenschule seines nahe gelegenen Geburtsortes Lobberich wechselte: ${ }^{4}$

Der Patron unseres Gymnasiums war der grosse Mystiker Thomas a Kempis, der Verfasser der Imitatio Christi, des nächst der Bibel meist verbreiteten und übersetzten Buchs der Weltli-

\footnotetext{
1 Keyser (1992).

2 Jaeger (1963) = Jaeger (1961).

3 Keyser (1992) 83-89.

4 Jaeger (1960b) X.

DOI 10.1515/9783110548983-009
} 
teratur. Die Stille und Innerlichkeit seiner Weltbetrachtung, die noch in der Frömmigkeit des Niederrheins zu spüren war, passte zu der Schönheit der heimischen Seenlandschaft.

Kempen gehörte seit napoleonischen Zeiten zu den preußischen Rheinlanden, aber die Mehrheit der Stadt war katholisch. Die Familie Jaeger dagegen war seit Generationen durch ihre evangelisch-lutherische Konfession geprägt und lebte am Niederrhein, wie Jaeger selbst in anfänglichen, aber ebenfalls späten Notizen für eine Autobiographie schreibt, „the age-old, deeply rooted legacy of their Protestant faith“. ${ }^{5}$ Freilich: Von einer gefestigten evangelischen Identität mit antikatholischer Spitze ist bei Jaeger weder in den späten autobiographischen Texten noch in anderen Veröffentlichungen (jedenfalls so weit ich sie kenne) etwas zu spüren; wenn Jaeger seine Mentalität in den späten autobiographischen Notizen als eine Synthese aus der Frömmigkeit seiner Großeltern und der Rationalität seiner Eltern beschreibt, wirkt dieser Dual nicht wenig konstruiert. In Wahrheit bilden schon die genannten Großeltern im Blick auf ihre religiösen Mentalitäten keine Einheit: Der Großvater Johann Ludwig Birschel wird von Jaeger als liberaler kaiserzeitlicher Protestant portraitiert: Er war „entirely non political and non church-going [and had] an enlighted outlook ", ${ }^{6}$ während die Großmutter Pauline Birschel offenbar vom rheinischen Pietismus in Wuppertal geprägt war, den Jaeger für ein englisches Publikum (historisch nicht vollständig zutreffend) als „a genuine theocracy, like those in the Puritan Commonwealths of New England where the Pastor ruled and theology penetrated all thought and earthly conduct ${ }^{\text {"7 }}$ beschreibt. Jaeger selbst charakterisiert sich in den letzten Schuljahren in jenem späten Essay als halben Theologen: „Even in the Gymnasium I was half a theologian, and my graduation certificate specified that I would go on to the study of both classical philology and theology." ${ }^{8}$ Rückschlüsse auf die persönliche Frömmigkeit von Werner Jaeger erlauben aber alle diese (meist, wie gesagt, sehr späten Zeugnisse) im Unterschied zu Annahmen von Paul Keyser nicht. Die spannende Frage, wie er mit seiner zweiten, jüdischen Frau zusammenlebte, vermag ich nicht zu beantworten; jedenfalls ist er nach der Ordnung eines memorial service beerdigt worden. ${ }^{9}$

Auch wenn wir so wenig über Jaegers persönliche Haltung zur Religion und seine Frömmigkeit wissen, scheint mir deutlich, dass die Äußerungen über die starke theologische Prägung während der Kempener Gymnasialzeit eher auf ein

\footnotetext{
5 Jaeger (1966) 11.

6 Jaeger (1966) 11.

7 Jaeger (1966) 15.

8 Jaeger (1966) 38.

9 Order of Funeral Service, Harvard University Memorial Church, 22.10.1961.
} 
wissenschaftliches Interesse am Christentum hindeuten als auf eine besonders intensive Religiosität. Denn spätestens beim Studium in Marburg und Berlin erfolgte die endgültige Entscheidung für die klassische Philologie. Laut der Vita, die der Dissertation beigefügt ist, studierte Jaeger allerdings auch bei Theologen (wobei es mir nicht gelungen ist aufzuhellen, bei welchen). ${ }^{10}$ Manches spricht für Harnack: William Calder III berichtet, dass im Büro Jaegers in Harvard „the twin icons“ von Wilamowitz und Harnack hingen; Wilamowitz war ohne Zweifel die prägende philologische Lehrgestalt des Berliner Studiums der Jahre 1907 bis 1911 und dann der Jahre bis zur Habilitation im Jahre 1914: „Die grösste Erscheinung im Leben der Philologie um die Jahrhundertwende war unstreitig Wilamowitz, sein Name traf unser Ohr schon im Schulunterricht, dessen geschichtlichen Horizont sein griechisches Lehrbuch zu erweitern suchte."11 Wenn man sich allerdings klarmacht, dass der Gräzist Wilamowitz seinen kirchenhistorischen Kollegen Harnack wegen angeblich mangelnder philologischer Kompetenz nicht sonderlich schätzte und zudem offenbar von tiefem Neid auf die engen Beziehungen zwischen dem Schwiegervater Mommsen und dessen liberalem Ersatzschwiegersohn Harnack erfüllt war, stellte das Bilderpaar im Büro eine durchaus kühne Nachbarschaft dar. Man kann sich angesichts der deutlichen Vorbehalte, die Wilamowitz in Briefen an Jaeger über den Schwiegervater Mommsen äußerte, ${ }^{12}$ schwer vorstellen, dass man gleichzeitig Wilamowitz und Harnack nahe stehen konnte. Gleichwohl spricht Jaeger einmal brieflich „,von dem persönlich Bestrickenden an Harnack“. ${ }^{13}$ Wie nahe sich Harnack und Jaeger wirklich standen, ist im Unterschied zu dem Verhältnis zwischen Wilamowitz und Jaeger - trotzdem schwer zu sagen; der Berliner Nachlass Harnacks überliefert keine Korrespondenz zwischen beiden und die große Biographie der Tochter erwähnt den gräzistischen Kollegen nicht. ${ }^{14}$ Manches spricht für eher lose Kontakte und leichte Distanz: Mitglied der berühmten Kirchenväterkommission der preußischen Akademie, die unter Harnacks Leitung die „Griechischen Christlichen Schriftsteller“ herausgab, wurde Jaeger erst im Jahre 1927 (gemeinsam mit Erich Klostermann [1870 - 1963]), drei Jahre vor Harnacks Tod. ${ }^{15}$ Im folgenden Jahr 1928 trat die Kommission erstmals wieder zusammen, nachdem sie seit 1924 wegen Mangels an Geldmitteln aufgrund der Inflation nicht mehr getagt hatte; Jaeger nahm an dieser Sitzung nicht teil und bei der geplanten Umgestaltung der traditionsreichen Kirchenvä-

10 Jaeger (1911) 61; der Text der Diss. ohne die Vita: Jaeger (1960a) 1-38; vgl. auch ders. (1966) 38.

11 Jaeger (1960b) XI.

12 Rebenich (1997) 235-237.

13 Calder (1983) 185 Anm. 105.

14 Zahn-Harnack (1936).

15 Harnack (2000) 159. 
terkommission in eine „Kommission zur Förderung der kirchlichen und religionsgeschichtlichen Studien im Rahmen der römischen Kaiserzeit“ sollte auch nicht der amtierende Ordinarius, sondern sein Vorgänger Wilamowitz-Moellendorff helfen, beim preußischen und Reichs-Finanzministerium weiteres Geld zu besorgen. ${ }^{16}$

Deutlich anders verhielten sich die Dinge offenbar zwischen Jaeger und einem anderen kaum weniger prominenten Berliner Kirchenhistoriker: Ein sehr freundlicher Brief an Harnacks Nachfolger, den Kirchenhistoriker Hans Lietzmann (1875-1942), den Jaeger an Bord der „New York“ am 29. November 1936 schreibt und der allerlei Eindrücke über die Lage in Oxford enthält, ${ }^{17}$ deutet darauf hin, dass beide Gelehrte sich besser verstanden als Harnack und Jaeger. Allerdings beschäftigen sich die erhaltenen Briefe mit dem Schicksal vom Berliner Unternehmen Jaegers nach dessen Emigration, mit der Zeitschrift „Antike“ und der Ausgabe der Werke Gregor von Nyssas, so dass hier auch interessengeleitete Höflichkeit im Spiel gewesen sein mag. Lietzmanns Freund, der damals längst in München wirkende Eduard Schwartz, bezeichnete den später in Tübingen lehrenden Berliner Altphilologen Jürgen Kroymann (1911-1980) wenig freundlich als eine „Jaegerkreatur“18 und nannte kurz darauf den Berliner Doktorvater Kroymanns im Blick auf seine Akademieabhandlung „Tyrtaios über die wahre APETH“19 „in der Hauptsache ein Blender ...; das Problem war von Wilamowitz richtiger gesehen. “20 Sicher ist, dass Jaeger und Lietzmann Aufsätze austauschten: Als Gegengabe zu einem Aufsatz Lietzmanns, der sich mit der Authentizität der Traditionen über ein römisches Martyrium des Apostels Petrus beschäftigte, ${ }^{21}$ sendete Jaeger den englischen Aufsatz über die ersten griechischen Berichte über die Juden; ${ }^{22}$ auf dieses Problem war er, wie er schreibt, durch sein Diokles-Buch gestoßen. ${ }^{23}$ Ein wunderschöner Brief Lietzmanns mit einer Lobeshymne für Jaegers Demosthenes aus dem Jahr 1940 schließt die erhaltene, aber sicher nur fragmentarisch überlieferte Korrespondenz ab; Lietzmann hofft in dem Brief auf „einen gerechten Frieden und wirkliche Freiheit“"24 - vergeblich, wie wir wissen: er starb kaum zwei Jahre später.

16 Harnack (2000) 160.

17 Jaeger an Lietzmann, 29.11.1936, in: Aland (1979) Nr. 975, 863-864.

18 Schwartz an Lietzmann, 19.03.1937, in: Aland (1979) Nr. 991, 875-876.

19 Jaeger (1932).

20 Schwartz an Lietzmann, 01.04.1937, in: Aland (1979) Nr. 994, 878-879.

21 Lietzmann (1936).

22 Jaeger (1938a).

23 Jaeger an Lietzmann, 19.07.1938, in: Aland (1979) Nr. 1051, 923-924. - Vgl. Jaeger (1938b).

24 Lietzmann an Jaeger, 19.07.1938, in: Aland (1979) Nr. 1135, 989-990. - Vgl. Jaeger (1939). 
Doch zurück zum jungen Berliner Studenten Werner Jaeger. Es spricht viel dafür, dass er schon sehr früh durch den verehrten Lehrer auf die Spur desjenigen antiken christlichen Theologen gesetzt wurde, der ihn zeitlebens beschäftigen sollte: Gregor von Nyssa, ${ }^{25}$ möglicherweise in einem Augenblick, als Jaeger nach der Dissertation im Jahre 1911 nicht genau wusste, welchem Autor er sich nun zuwenden solle. ${ }^{26}$ Drei Jahre später erschien die Habilitation über den spätantiken Bischof Nemesius von Emesa, ${ }^{27}$ im Vorwort ist berichtet, dass schon 1911 in Rom ein Zufall und seine „handschriftlichen Untersuchungen zur Vorbereitung der kritischen Erstausgabe des Gregor von Nyssa zu Nemesios geführt hatten“. ${ }^{28}$ Blicke auf das antike Christentum enthält das kleine Büchlein praktisch nicht, sondern, wie der Titel verspricht, Bemerkungen über Poseidonius, Galen und den älteren Neuplatonismus.

Jaeger hat immer wieder gesagt, dass er zum Studium der Texte antiker christlicher Autoren durch die textkritische Arbeit gekommen sei - also (und diese Selbstaussage muss man ernst nehmen) nicht durch seine schon früh ausgeprägten Interessen an einer Geistesgeschichte des Platonismus. Das wird zunächst einmal deutlich in der Antrittsrede, die Jaeger nach der Zuwahl in die Preußische Akademie der Wissenschaften am 17. Januar 1924, drei Jahre nach der Berufung als Nachfolger von Wilamowitz an die Berliner Friedrich-Wilhelms-Universität, ${ }^{29} \mathrm{am}$ Leibniztag (3. Juli 1924) hielt. Dabei sagte er: ${ }^{30}$

Meine textkritische Arbeit, die einer seit früher Jugend betätigten Neigung zu sprachlichen, wort- und stilgeschichtlichen Untersuchungen entspringt, empfing, wie ich mich deutlich erinnere, einen entscheidenden Impuls durch die unerwartete handschriftliche Bestätigung mehrerer Emendationen, die ich Diels als junger Student in einer Preisarbeit vorlegte. Meine Ausgaben mehrerer aristotelischer Schriften, von denen die der Metaphysik noch ungedruckt liegt, sowie der beiden ersten Bände des Kirchenvaters Gregor von Nyssa führten mich mehrfach und z.T. für längere Zeit an die Stätten der antiken Kultur und erfüllten mich mit realer Anschauung des historischen Lebens und jeglicher Art der Überlieferung, besonders der handschriftlichen Geschichte der Texte. So etwas lernt man nicht, wenn man es nicht selbst gemacht hat, und trotz anders gerichteter moderner Meinungen gestehe ich, daß ich mir den Philologen ohne dies enge Verhältnis zu den Texten und den Problemen der Überlieferung nicht zu denken vermöchte.

25 Jaeger (1966) 38.

26 Keyser (1992) 86 Anm. 28.

27 Jaeger (1914).

28 Jaeger (1914) V.

29 Vgl. den Zuwahlvorschlag von Wilamowitz, den E. Norden, W. Schulze, E. Meyer, K. Holl, U. Stutz, Th. Wiegand, H. Maier u.U. Wilcken zeichnen, bei: Kirsten (1985) 176.

30 Jaeger (1924) LXXXVII. 
Interessanterweise wird die Edition der Werke des Gregor von Nyssa einfach bei den Akademie-Mitgliedern als bekannt vorausgesetzt und zunächst nicht näher erläutert. Es handelt sich um die beiden Bände des Werks Contra Eunomium, die in erster Auflage 1920 mit einem Vorwort des Lehrers Wilamowitz erschienen und die von Jaeger herausgegebene Reihe der Gregorii Nysseni Opera begründeten, die inzwischen über fünfzehn Bände umfasst und immer noch nicht abgeschlossen ist. ${ }^{31}$ In der Berliner Kirchenväterausgabe der Preußischen Akademie, den von Harnack begründeten „Griechischen Christlichen Schriftstellern“, konnten die Werke Gregors nicht erscheinen, weil diese Reihe den Schriftstellern der ersten drei Jahrhunderte vorbehalten war, Gregor aber im vierten Jahrhundert lebte. Im Unterschied zu Wilamowitz und Jaeger hielt Harnack die vornizänische Epoche des antiken Christentums für die maßgebende „paläoontologische Schicht“ der neuen Religion, die zentrale Phase einer ebenso problematischen wie unvermeidlichen „Hellenisierung“ des Christentums. ${ }^{32}$ Der Unterschied zu den Bänden der Berliner Kirchenväterausgabe Harnacks ist mit Händen zu greifen; die Sprache der praefationes und des Apparates ist bei der Gregor-Ausgabe lateinisch, bei den „Griechischen Christlichen Schriftstellern“ bestand Harnack auf dem von ihm als modern empfundenen Deutsch. ${ }^{33}$ Wilamowitz stellte zur Finanzierung der GregorEdition Mittel einer Sammlung zu Ehren seines sechzigsten Geburtstags zur Verfügung. Jaeger hat in seiner späten Einleitung zu den „Scripta minora“ beschrieben, was diese Aufgabe von anderen editorischen Projekten unterschied: ${ }^{34}$

\footnotetext{
Wegen ihrer reichen handschriftlichen Tradition haben die Kirchenväter mich als Philologen auch abgesehen von ihrem inhaltlichen Interesse stets angezogen. Als Herausgeber des Gregor von Nyssa, dessen zahlreiche Schriften in weit über tausend Handschriften überliefert sind, sah ich mich vor ganz neue Aufgaben gestellt, wie die klassische Philologie sie bisher kaum in Angriff genommen hatte. Darüber berichten die ausführlichen Prolegomena der einzelnen Bände. Es zeigte sich, dass auch bei der reichsten Überlieferung eines Textes mit der Methode der recensio allein nicht auszukommen ist und dass der emendatio darüber hinaus stets ihr Platz erhalten bleibt, zumal bei Texten, die wie die der christlichen Väter nicht durch Grammatikerhände gegangen und dadurch normalisiert sind. Die Kunst der Emendation schien in der Philologie unserer Tage weitgehend verloren gegangen zu sein, teils weil das Beste durch frühere Generationen schon vorweggenommen war, teils aus Unbegabtheit für diese Dinge und abergläubischer Buchstabentreue, die sich für gesunden Konservatismus hält.
}

31 Hörner (1971).

32 Markschies (2009) 538-549 sowie ders. (2012) 48-58.

33 Markschies (2004).

34 Jaeger (1960b) XVIf. 
Aber natürlich interessierte sich Jaeger nicht nur deswegen, weil die Textkritik bei den Werken der spätantiken Kirchenväter spannender war als bei denen klassischer Schriftstellern, für Autoren wie Nemesius von Emesa oder Gregor von Nyssa. Selbstverständlich reizten ihn angesichts seines Interesses für die Geistesgeschichte des Platonismus auch die Inhalte. Auch das zeigt die erwähnte Antrittsrede als Mitglied der Preußischen Akademie der Wissenschaften am Leibniztag 1924, allerdings auf recht besondere Weise. Es gehört zwar zum Genre solcher Antrittsreden, neben einer Erläuterung des wissenschaftlichen Werdeganges und einer Reverenz für die akademischen Lehrer die Programmatik künftiger Jahre mit klaren Worten zu entwickeln. Doch Jaeger nahm so offenkundig gegen Harnack und dessen Programm einer Edition vornizänischer Kirchenväter durch Theologen Stellung, dass diese Frontstellung jedem auffallen musste, der einigermaßen über die Tätigkeiten der Akademie orientiert war. Jaeger sagte: ${ }^{35}$

Das Studium der Kirchenväter und der Dogmengeschichte führte mich auch inhaltlich tief in die altchristliche Welt und in ihre Zusammenhänge mit dem Altertum hinein. Ein von jeher reges Interesse für alle Formen und Äußerungen des Religiösen, das bei mir nicht wie bei den meisten Philologen vor dem Christentum haltmachte, verband sich mit der Liebe zur antiken Philosophie und reizte mich, der geistigen Kontinuität zwischen antikem und christlichem Denken nachzugehen. Der Ausgangspunkt zu meinem Büchlein Nemesios von Emesa war der Umstand, daß das anthropologische Werk dieses christlichen Bischofs, eine wichtige Quelle mittelalterlicher Philosophie, zum Teil unter dem Namen des Gregor von Nyssa überliefert ist. Die Erforschung der Nachwirkung antiker Weltanschauung und Philosophie in der christlichen Spätantike steht noch in den Anfängen. Sie stand bisher zu ausschließlich unter dem Zeichen der theologischen Dogmengeschichte und der philologischen Jagd nach Quellen und Fragmenten. Wenn die Ausbeutung der christlichen Literatur nach dieser Richtung auch noch große Überraschungen verspricht, so ist es doch an der Zeit, ein mehr organisches Verständnis jenes großen geistigen Prozesses anzubahnen und die durch das Christentum bewirkte Umlagerung der inneren Struktur der antiken Welt als eine große geschichtliche Aufgabe der Altertumswissenschaft ins Auge zu fassen, in die wir uns mit den Theologen teilen müssen.

Auch wenn hier kein Name fällt, ist deutlich, wer im Blick ist, wenn Jaeger davon spricht, dass die „Erforschung der Nachwirkung antiker Weltanschauung und Philosophie in der christlichen Spätantike“ bislang „zu ausschließlich unter dem Zeichen der theologischen Dogmengeschichte und der philologischen Jagd nach Quellen und Fragmenten“ gestanden habe - gemeint ist natürlich Harnack, dessen Bild der christlichen Antike ganz stark von seinem Konzept einer Dog-

35 Jaeger (1924) LXXXVII-LXXXVIII. 
mengeschichte (wie es im Motto seines magistralen Werks heißt) ${ }^{36}$ als einer Kritik des Dogmas bestimmt war. Da Harnack als Maßstab seiner Kritik das angeblich dogmenfreie Urchristentum heranzog und die ebenso angeblich schlichte fromme Botschaft Jesu über den Vater, interessierte ihn (mit einem zeitgenössischen Terminus gesprochen) die Rezeptions- oder besser Transformationsgeschichte antiker Philosophumena im antiken Christentum im Grunde nur als Verfallsgeschichte. Der Vorwurf, bisher sei zu viel der philologischen Jagd nach Quellen und Fragmenten geschehen, den Jaeger gleichfalls in seiner in Wahrheit höchst polemischen Antrittsrede erhebt, trifft ebenfalls Harnack und sein großes Inventarwerk „Geschichte der altchristlichen Literatur“, das als Quellen- und Fragmentensammlung der Ausgabe der „Griechischen Christlichen Schriftsteller“ vorausging. ${ }^{37}$ Nun könnte man denken, antike christliche Autoren seien für Jaeger nur deswegen interessant, weil sie platonisches und neuplatonisches Denken rezipieren. Doch dem ist nicht so. In seinen späten einführenden Bemerkungen zu den „Scripta Minora“ beschreibt Jaeger diese Zusammenhänge folgendermaßen: ${ }^{38}$

Die Einheit (discordia concors) klassischer und spätantik-christlicher Geschichte war mir, wie gesagt, als selbstverständlich überliefert, und der Weg von der Antike zur christlichen Religion war mir seit meiner Kindheit natürlich. Ich wurde auf katholischen Schulen gross, die für mich keine religiöse Belehrung boten, denn ich war protestantisch, obwohl in einem religiös nicht positiv interessierten Elternhause aufgewachsen. Ich musste mir daher die Einsicht in die Ursprünge des Christentums grossenteils selbst erwerben. Ich tat dies, wie ich es mit der Antike gemacht hatte, durch das fortgesetzte Eindringen in die Quellen. So war ich auf dem Gymnasium schon ein halber Theologe, und in meinem Abiturientenzeugnis hiess es denn auch, dass ich entlassen würde zum Studium der klassischen Philologie und der Theologie. Auf der Universität führte ich meine theologischen Studien in der Tat ständig weiter, aber in derselben Weise wie vorher, nämlich privatim durch Lektüre der altchristlichen wie der modernen theologischen Literatur. Theologische Vorlesungen habe ich freilich nur wenige gehört. Doch verfolgte ich die religiösen Bewegungen meiner Zeit mit tiefer Anteilnahme und war in der Kirche aller Jahrhunderte zu Hause, ohne aktiv im Leben irgend einer Kirche darinzustehen. Der geschichtliche Weg zu dem jüdisch-christlichen Erbe war der mir gemässe. Ich sah ein gleiches, wenn auch in verschiedenem Grade, bei Philologen wie P. Wendland, Ed. Schwartz u. a. sich vollziehen. Daher fühlte ich mich sogleich in meinem Element, als mich Wilamowitz nach meinem Berliner Doktorexamen für den Gedanken zu gewinnen suchte, an der Herausgabe des Gregor von Nyssa einen führenden Anteil zu nehmen.

36 Harnack (1909) passim; vgl. Basse (2001) 123-146.

37 Harnack (1893); ders. (1958).

38 Jaeger (1960b) XXIIf. 
Es geht also nicht um eine schlichte Rezeptions- (oder meinetwegen auch: Transformations-) Beziehung zwischen paganem und christlichem Erbe, sondern gleichsam in angewandter platonischer Philosophie um „die Einheit (discordia concors)“ zwischen zwei als Entitäten gedachten geistesgeschichtlichen Phänomenen. Wie früh sich diese Orientierung auf solche Linien im Werk Werner Jaegers zeigt, wird deutlich, wenn man die umfangreiche Rezension zu Eduard Nordens Werk „Agnostos Theos“ zur Hand nimmt, die er 1913 veröffentlicht hat, ein Jahr vor seiner Berliner Habilitation. ${ }^{39}$ Da Keyser sie ausführlich besprochen hat, ${ }^{40}$ können wir es in unserem Zusammenhang bei einem Hinweis belassen. Keyser macht auch auf die Reste eines Briefwechsels zwischen Jaeger und dem Marburger Neutestamentler Rudolf Bultmann (1884-1976) aufmerksam, die sich im Besitz von William Calder III befinden. ${ }^{41}$

In den folgenden Jahren hat Werner Jaeger sich bei der Beschäftigung mit spätantiken christlichen Theologen vor allem auf Gregor von Nyssa konzentriert. Wenn er beispielsweise in den Sitzungsberichten der Preußischen Akademie der Wissenschaften einen „neuentdeckten Kommentar zum Johannesevangelium“ behandelt, dann hat ihn offenbar länger die Frage beschäftigt, ob Gregor der Autor des Textes ist, und er teilt nun mit, warum er diese Position zwar erwogen, aber nun verworfen hat. ${ }^{42}$

In den Jahren 1952 und 1953 beschäftigte sich Jaeger in Harvard nochmals mit der Edition dreier asketischer Schriften des Gregor, De instituto Christiano, De professione Christiana sowie De perfectione. ${ }^{43}$ Dabei fiel ihm auf, dass die erste Schrift Gregors nahe Verwandtschaft zu einem Text aufweist, den der Göttinger Kirchenhistoriker Hermann Dörries (1895-1977) entdeckt hatte, der Epistula magna des Pseudo-Macarius/Symeon. Die drei Schriften Gregors erschienen im ersten Teil des achten Bandes der Werkausgabe Gregors. Das im folgenden Jahr 1953 als Supplement der Reihe veröffentlichte Buch „Two Rediscovered Works of Ancient Christian Literature: Gregory of Nyssa and Macarius“ aus dem Jahre 1953 enthält nicht nur einen vorläufigen Text der Epistula magna, den die Überlieferung einem Asketen namens Macarius, dem Ägypter, (fälschlicherweise) zuschreibt, sondern eine Untersuchung der literarischen Beziehungen dieses Briefs zur Schrift De instituto Christiano des Gregor von Nyssa. Diese Schrift, mit einem deutschen Titel „Über das gottgewollte Ziel und die wahre Askese“, hatte Jaeger, wie gesagt, im Jahr zuvor als achten Band seiner Gregor-Ausgabe ediert. Die Epistula magna

39 Jaeger (1913) = ders. (1960a) 115-161.

40 Keyser (1992) 89-90.

41 Keyser (1992) 90-92.

42 Jaeger (1930) 584-585.

43 Jaeger (1952) 1-89, 91-142, 143-214. 
erschien ihm als eine Bearbeitung des Traktates von Gregor; heute ist deutlich, dass man die literarischen Beziehungen genau umgekehrt rekonstruieren muss: Gregor bearbeitete in Wahrheit den großen Brief. Es ist sicher nicht zu viel psychologisierende Interpretation Werner Jaegers, wenn man vermutet, dass die Begeisterung über das Werk Gregors den nun in Amerika lebenden Gräzisten dazu führte, die Abhängigkeitsrelationen (jedenfalls nach unserer heutigen Perspektive) genau falsch herum zu rekonstruieren. So hat es jedenfalls Reinhart Staats vor nunmehr dreißig Jahren im Vorwort seiner Neu-Edition des großen Briefes formuliert: ${ }^{44}$

Jaeger hatte gemeint, daß dem großen Kappadozier mit De instituto Christiano als einem reifen Alterswerk die von ihm angestrebte Synthese von Antike und Christentum besonders glücklich gelungen war. Jaeger ... liebte nächst Gregor von Nyssa kaum einen Theologen so sehr wie den Humanisten Erasmus von Rotterdam. Hier wie dort fand er das Zusammenwirken von Gnade und Werk, den klassischen Synergismus, den Jaeger tief bejahte, er selbst war ja ein Gegner von Augustins und Luthers Gnadenlehre. Was Theologen wie Adolf von Harnack als Hellenisierung der christlichen Religion abwerteten, bekam bei Werner Jaeger eine positive Deutung. Statt von einer Hellenisierung des Christentums sprach er von Übernahme eines Leitmotivs seines Hauptwerkes ,Paideia‘ ... von der ,Paideia Christi‘.

Ausgeführt hat Werner Jaeger diese Gedanken in seinem letzten, bereits erwähnten Buch, mit dem wir auch schließen wollen - in den Carl Newell Jackson-Vorlesungen, die Jaeger 1960 unter dem Titel „Early Christianity and Greek Paideia“ in Harvard hielt. Sie wurden auf Anregung seines damals in Tübingen als Kirchenhistoriker tätigen Berliner Schülers Walther Eltester (1899-1976) ${ }^{45}$ ins Deutsche übersetzt, Eltester besorgte selbst die Übertragung und die Vorlesungen erschienen 1963 unter dem Titel „Das frühe Christentum und die griechische Bildung“ im Verlag Walter de Gruyter in Berlin. ${ }^{46}$ Paul T. Keyser hat dieses Büchlein sehr pointiert in seinem Beitrag für das Kolloquium „Werner Jaeger reconsidered“ „not as merely volume four of Paideia (or worse, as merely an afterthought) but as the telos or entelechy of the whole of not only Paideia but of Jaeger's life “ bezeichnet. ${ }^{47}$ Aber kann man bei einer „Einheit (discordia concors) klassischer und spätantikchristlicher Geschichte“, die Jaeger noch einmal in der Einleitung seiner gesammelten Schriften aus dem Jahre 1960 beschwört, wirklich von einer derartigen Entelechie in Richtung einer der beiden Pole der Einheit sprechen? Oder wird die spannungsvolle Einheit der Gegensätze bei solcher Interpretation nicht in eine

\footnotetext{
44 Staats (1984) 11-12.

45 Andresen (1977) 1.

46 Jaeger $(1963)=$ ders. (1961).

47 Keyser (1992) 89.
} 
große Denkbewegung hin in den erneuerten christlichen Platonismus aufgehoben, mithin mehr Beierwaltes ${ }^{48}$ als Jaeger in dieser Interpretation präsentiert? Im Jahr 1930 schreibt Jaeger an Bultmann, dass das Christentum zwar in die Antike eintreten und sie durchdringen konnte, aber sie nicht überwinden und durch einen anderen Aufbau zu ersetzen vermochte. ${ }^{49}$ Eine Entelechie besteht nach der Aussage dieses Briefes also nicht in Richtung des Christentums, sondern allenfalls in Richtung der Antike. Da diese aber durch das Christentum transformiert ist, gilt zugleich eben das Wort von der Einheit der Gegensätze, der discordia concors.

Der Autor der Vorlesungen unter dem Titel „Early Christianity and Greek Paideia“ (oder eben deutsch „Das frühe Christentum und die griechische Bildung“) beweist gleich auf den ersten Seiten seine gute Kenntnis der zeitgenössischen Debatten innerhalb der theologischen Wissenschaft, wenn er festhält, dass Religion und Kultur entgegen der Positionen von Karl Barth und Emil Brunner nicht separiert werden können wie dürfen. ${ }^{50}$ Eine ausführliche Würdigung dieses Werks ist in unserem Rahmen nicht notwendig; auch hier hat Keyser wichtige Vorarbeiten geleistet. Trotz einer chronologisch vom Neuen Testament über den ersten Clemensbrief, die Apologeten, die christlichen Alexandriner Clemens und Origenes sowie Gregor von Nyssa geordneten Abfolge bleiben Lücken. Jaeger war sich bewusst, dass er mit seinen Veröffentlichungen und den Carl Newell JacksonVorlesungen in Harvard 1960 die Brücke vom antiken zum christlichen Denken noch nicht vollständig geschlagen hatte. In der Einführung in den ersten Band seiner „Scripta Minora“ schreibt er wenig später: ${ }^{51}$

Die in meinem Buch über die Theologie der frühgriechischen Denker verheissene Ausfüllung der Lücke zwischen der frühgriechisch-vorsokratischen und der platonisch-christlichen Theologie eines Origenes und Clemens bleibt eine wichtige Aufgabe der Zukunft.

So viel Selbstrelativierung beeindruckt, da der, der sich dort relativiert, dies auf einem relativ hohen Niveau vollzieht. Denn er ist mit den christlichen Autoren, die er als Beispiele seines Rezeptions- und Transformationsprozesses analysiert, wohl vertraut. Beispielsweise mit dem ersten Clemensbrief, einem Gemeindebrief der stadtrömischen Christenheit an die Schwestergemeinde in Korinth vom Ende des ersten Jahrhunderts. Werner Jaeger hat sich einmal in einem bereits erwähnten Brief an den Berliner Kirchenhistoriker Hans Lietzmann aus dem Jahre 1938 „als

\footnotetext{
48 Beierwaltes (2014).

49 Keyser (1992) 91-92.

50 Jaeger (1963) 1.

51 Jaeger (1960b) XXII.
} 
alter Clemensfreund“ bezeichnet;5 man wird angesichts der einschlägigen Passagen in „Early Christianity and Greek Paideia“ nicht widersprechen wollen.

Die Zeiten der großen Kultursynthesen und der großen Konzepte zum Thema „Antike und Christentum“ sind unwiederbringlich vorbei; „Christentum ist auch Antike“ hat Jacques Fontaine ${ }^{53}$ vor einiger Zeit pointiert formuliert, um das Problem derartiger Vergleiche zwischen zwei scheinbar stabilen Entitäten aufzuspießen. Aber Werner Jaeger sah sich nicht nur aufgrund seines Interesses am Fortwirken des Platonismus zum antiken Christentum gedrängt, sondern auch wegen seiner Leidenschaft für die Textkritik ganz bestimmter Überlieferungen. Vor unserem geistigen Auge erscheinen die imponierenden Bände der Werkeausgabe Gregor von Nyssas und das Lexicon Gregorianum - und plötzlich wird deutlich: Hier ist nun wirklich nicht alles vergangen, ganz im Gegenteil.

\section{Literaturverzeichnis}

Aland (1979): Kurt Aland (Hg.), Glanz und Niedergang der deutschen Universität. 50 Jahre deutscher Wissenschaftsgeschichte in Briefen von und an Hans Lietzmann (1892-1942), mit einer einführenden Darstellung, Berlin/New York.

Andresen (1977): Carl Andresen, „Nachruf auf Walther Eltester“, Zeitschrift für die neutestamentliche Wissenschaft 68, 1.

Basse (2001): Michael Basse, Die dogmengeschichtlichen Konzeptionen Adolf von Harnacks und Reinhold Seebergs, Forschungen zur Kirchen- und Dogmengeschichte 82, Göttingen.

Beierwaltes (2014): Werner Beierwaltes, Platonismus im Christentum (Philosophische Abhandlungen 73), 3., erweiterte Aufl., Frankfurt/Main: Vittorio Klostermann.

Calder (1983): William M. Calder III, Ulrich von Wilamowitz-Moellendorff, Selected Correspondence 1869-1931, Antiqua 23, Neapel.

Fontaine (1982): Jacques Fontaine, „Christentum ist auch Antike. Einige Überlegungen zu Bildung und Literatur in der lateinischen Spätantike“, Jahrbuch für Antike und Christentum 25 (1982) 5-21

Harnack (1893): Adolf von Harnack, Geschichte der altchristlichen Litteratur bis Eusebius, 1.TI. Die Überlieferung und der Bestand der altchristlichen Litteratur bis Eusebius, bearb. unter Mitwirkung v. Erwin Preuschen, Leipzig.

Harnack (1909): Adolf von Harnack, Lehrbuch der Dogmengeschichte. Bd. 1 Die Entstehung des kirchlichen Dogmas, Sammlung Theologischer Lehrbücher 2/1, Tübingen.

Harnack (1958): Adolf von Harnack, Geschichte der altchristlichen Literatur bis Eusebius, 2.TI. Die Chronologie der altchristlichen Litteratur, 2 Bde., Leipzig 1896/1904 (= Leipzig 1958).

Harnack (2000): Adolf von Harnack, Protokollbuch der Kirchenväter-Kommission der Preußischen Akademie der Wissenschaften 1897-1928, Diplomatische Umschrift von

52 Jaeger an Lietzmann, 19.07.1938, in: Aland (1979) Nr. 1051, 923.

53 Fontaine (1982) 5-21 sowie Markschies (2006) 17-34. 
Stefan Rebenich, Einleitung und kommentierende Anmerkungen von Christoph Markschies, Berlin/New York.

Hörner (1971): Hadwiga Hörner, „Über Genese und derzeitigen Stand der grossen Edition der Werke Gregors von Nyssa“, in: Marguerite Harl (Hg.), Écriture et culture philosophique dans la Pensée de Grégoire de Nysse. Actes du Colloque de Chevetogne (22-26 Septembre 1969), Leiden, 18-50.

Jaeger (1911): Werner Jaeger, Emendationum Aristotelearum Specimen, Diss. Berlin. Jaeger (1913): Werner Jaeger, Rez. „Eduard Norden, Agnostos Theos. Untersuchungen zur Formengeschichte religiöser Rede, Leipzig: B.G. Teubner, 1913“, Göttingische Gelehrte Anzeigen 1913, 569-610.

Jaeger (1914): Werner Jaeger, Nemesios von Emesa. Quellenforschungen zum Neuplatonismus und seinen Anfängen bei Poseidonius, Berlin.

Jaeger (1924): „Antrittsrede des Hrn. Jaeger“, Sitzungsberichte der Preußischen Akademie der Wissenschaften, phil.-hist. Klasse 15 (1924), Berlin, LXXXVI-LXXXVIII.

Jaeger (1930): Werner Jaeger, „Der neuentdeckte Kommentar zum Johannesevangelium und

Dionysios Areopagites“, Sitzungsberichte der Preußischen Akademie der Wissenschaften 1930, 569-594.

Jaeger (1932): Werner Jaeger, „Tyrtaios über die wahre APETH“, Sitzungsberichte der

Preußischen Akademie der Wissenschaften, phil.-hist. Klasse 23 (1932), Berlin, 537-568. Jaeger (1938a): Werner Jaeger, „Greeks and Jews. The First Greek Records of Jewish Religion and Civilization“, The Journal of Religion 18, 127-143.

Jaeger (1938b): Werner Jaeger, Diokles von Karystos. Die griechische Medizin und die Schule des Aristoteles, Berlin.

Jaeger (1939): Werner Jaeger, Demosthenes. Der Staatsmann und sein Werden, Berlin. Jaeger (1952): Wernerus Jaeger, Johannes P. Cavarnos, Virginia Woods Callahan (Hgg.), Gregorii Nysseni Opera Ascetica, Leiden.

Jaeger (1960a): Werner Jaeger, Scripta Minora Vol. I, Rom.

Jaeger (1960b): Werner Jaeger, „Zur Einführung“, in: Jaeger (1960a) IX-XXVIII.

Jaeger (1961): Werner Jaeger, Early Christianity and Greek Paideia, Cambridge, MA.

Jaeger (1963): Werner Jaeger, Das frühe Christentum und die griechische Bildung, übers. v. Walther Eltester, Berlin.

Jaeger (1966): Werner Jaeger, „Notes towards an Autobiography: The Beginnings“, in: ders., Five Essays, transl. by Adele M. Fiske, Montreal, 3-21.

Keyser (1992): Paul T. Keyser, „Werner Jaeger’s Early Christianity and Greek Paideia“, in: William Calder III (Hg.), Werner Jaeger reconsidered (Proceedings of the second Oldfather conference, held on the Campus of the University of Illinois at Urbana-Champaign, April 26-28, 1990), Atlanta, 83-105.

Kirsten (1985): Christa Kirsten (Hg.), Die Altertumswissenschaften an der Berliner Akademie. Wahlvorschläge zur Aufnahme von Mitgliedern von F. A. Wolf bis zu G. Rodenwaldt 1799-1932, bearb. v. Herta Battré u. Ilse Neßler, mit einer Einführung von Heinz Kreißig †, Studien zur Geschichte der Akademie der Wissenschaften der DDR 5, Berlin.

Lietzmann (1936): Hans Lietzmann, „Petrus - römischer Märtyrer“, Sitzungsberichte der Preußischen Akademie der Wissenschaften, phil.-hist. Klasse 29 (1936), Berlin, 392 - 410. Markschies (2004): Christoph Markschies, „Das Problem der praefationes“, Zeitschrift für Antikes Christentum 8, 38-58. 
Markschies (2006): Christoph Markschies, „Antiquity and Christianity or: The Unavoidability of False Questions“, in: Beyond Reception. Mutual Influences between Antique Religion, Judaism, and Early Christianity, ed. by David Brakke, Anders-Christian Jacobsen, Jörg Ulrich, Early Christianity in the Context of Antiquity, 1, Frankfurt/Main, 17-34.

Markschies (2009): Christoph Markschies, „Adolf von Harnack. Vom Großbetrieb der Wissenschaft“, in: Annette M. Baertschi u. Colin G. King (Hgg.), Die modernen Väter der Antike. Die Entwicklung der Altertumswissenschaften an Akademie und Universität im Berlin des 19. Jahrhunderts, Transformationen der Antike 3, Berlin/New York, 529-552.

Markschies (2012): Christoph Markschies, Hellenisierung des Christentums. Sinn und Unsinn einer historischen Deutungskategorie, Theologische Literaturzeitung. Forum 25, Leipzig.

Rebenich (1997): Stefan Rebenich, Theodor Mommsen und Adolf Harnack. Wissenschaft und Politik im Berlin des ausgehenden 19. Jahrhunderts. Mit einem Anhang: Edition und Kommentierung des Briefwechsels, Berlin/New York.

Staats (1984): Reinhart Staats (Hg.), Makarios-Symeon, Epistola Magna. Eine messalianische Mönchsregel und ihre Umschrift in Gregors von Nyssa „De instituto christiano“, Abhandlungen der Akademie der Wissenschaften in Göttingen, phil.-hist. Klasse 3. Folge Nr. 134, Göttingen.

Zahn-Harnack (1936): Agnes von Zahn-Harnack, Adolf von Harnack, Berlin-Tempelhof. 